\title{
Study of the conformal region of the SU(3) gauge theory with domain-wall fermions
}

\author{
Junichi Noaki* \\ KEK High Energy Accelerator Research Organization \\ E-mail: noakiepost.kek.jp \\ Guido Cossu \\ KEK High Energy Accelerator Research Organization \\ E-mail: cossudpost.kek.jp \\ Ken-Ichi Ishikawa \\ Hiroshima University, Department of Physical Science \\ E-mail: ishikawadtheo.phys.sci.hiroshima-u.ac.ip \\ Yoichi Iwasaki \\ University of Tsukuba \\ E-mail: iwasakieccs.tsukuba.ac.jp \\ Tomoteru Yoshié \\ University of Tsukuba \\ E-mail: voshiedccs.tsukuba.ac.ip
}

\begin{abstract}
We investigate the phase structure of the SU(3) gauge theory with $N_{f}=8$ by numerical simulations employing the massless Domain-Wall fermions. Our aim is to study directly the massless quark region, since it is the most important region to clarify the properties of conformal theories. When the number of flavor is within the conformal window, it is claimed recently with Wilson quarks that there is the conformal region at the small quark mass region in the parameter space in addition to the confining phase and the deconfining phase. We study the properties of the conformal region investing the spatial Polyakov loops and the temporal meson propagators. Our data imply that there is the conformal region, and a phase transition between the confining phase and the conformal region takes place. These results are consistent with the claim that the conformal window is between 7 and 16. Progress reports on other related studies are also presented.
\end{abstract}

The 33rd International Symposium on Lattice Field Theory

14 - 18 July 2015

Kobe International Conference Center, Kobe, Japan

\footnotetext{
* Speaker.
} 


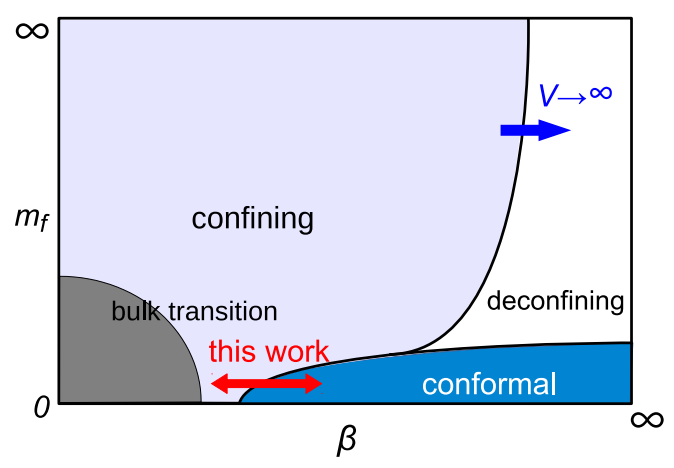

Figure 1: Sketch of the phase structure in the $\left(\beta, m_{f}\right)$-plane.

\section{Introduction}

The $S U(3)$ gauge theories with $N_{f}$-fermions in the fundamental representation which we call "many-flavor conformal QCD" are believed to have conformal properties when $N_{f}$ is within the conformal window. This model have been intensively studied in the context of the composite Higgs model as a candidate of the beyond standard model (See for a review [四].) Besides the phenomenological interest, it is theoretically interesting and important to clarify the properties of the theory. In refs. [, B] the authors claim, based on the study with the Wilson fermions, that

1. the conformal window is $7 \leq N_{f} \leq 16$

2. in addition to the confining phase and the deconfining phase there is a conformal region for $m_{f} \leq \Lambda_{\mathrm{IR}}$ (the IR-cutoff) in the deconfining phase

3. in the conformal region the vacuum is the non-trivial twisted $Z(3)$ vacuum modified nonperturbatively

4. in the conformal region meson propagators exhibit power corrected Yukawa type decay

Our objective is in this article to investigate these claims by numerical simulations with the massless $N_{f}=8$ Domain-Wall fermions. For this purpose, the phase diagram predicted in ref. [3]] is translated to the one shown in Figure 1, where $m_{f}$ is the fermion mass. In the light fermion region of the diagram, the conformal phase is separated from the confining phase at a certain value of $\beta$. On the other hand, in the heavier mass region, the deconfining phase would disappear in the limit of infinite lattice volume. The use of massless Domain-Wall fermions allows us to study the light mass region without tuning the fermion mass to zero as in the Wilson formalism. The fact that the chiral symmetry is realized to a good approximation is also advantageous to address the question whether or not the conformal theory is chiral symmetric.

After presenting the details of our numerical simulations in Section 2, we show, in Section 3, the vacuum structure based on the calculation of the spatial Polyakov loops and the meson temporal propagators. Section 4 contains progress report on the study of identifying the location of the IR fixed point using a scaling properties of meson propagators. We summarize our future plan in Section 5. 


\begin{tabular}{rrrrl}
\multicolumn{6}{l}{$V=8^{3} \times 32$} \\
\hline$\beta$ & $\Delta \tau$ & \#traj. & \multicolumn{1}{c}{ AC } & am $m_{\text {res }}$ \\
\hline \hline 2.0 & 0.25 & 8,000 & 500 & $2.2 \times 10^{-4}$ \\
2.3 & 0.25 & 12,000 & 1,000 & $2.7 \times 10^{-5}$ \\
2.6 & 0.10 & 11,000 & 2,500 & $1.2 \times 10^{-5}$ \\
4.2 & 0.25 & 10,000 & 1,500 & $1.3 \times 10^{-6}$ \\
4.7 & 0.25 & 9,000 & 1,500 & $1.2 \times 10^{-6}$ \\
6.0 & 0.25 & 9,000 & 1,500 & $8.9 \times 10^{-7}$ \\
\hline
\end{tabular}

\begin{tabular}{rrrrc}
\multicolumn{6}{l}{$V=16^{3} \times 64$} \\
\hline$\beta$ & $\Delta \tau$ & \#traj. & \multicolumn{1}{c}{ AC } & $a_{\text {res }}$ \\
\hline \hline 2.6 & 0.100 & 5,500 & 500 & $1.0 \times 10^{-5}$ \\
3.4 & 0.125 & 8,000 & 1,000 & $2.8 \times 10^{-6}$ \\
4.2 & 0.125 & 12,000 & 1,200 & $1.5 \times 10^{-6}$ \\
4.7 & 0.125 & 11,000 & 1,500 & $1.3 \times 10^{-6}$ \\
6.0 & 0.125 & 11,000 & 1,500 & $1.0 \times 10^{-6}$ \\
\hline
\end{tabular}

Table 1: Profiles of the generated gauge ensembles. For the lattice volume $V=8^{3} \times 32$ (left) and $16^{3} \times 64$ (right), values of $\beta$, MD-step size $\Delta \tau$, numbers of thermalized trajectories, a rough estimation of the autocorrelation (AC) time and typical values of the residual fermion mass are listed for each.

\section{Numerical Simulation}

In our numerical simulation, we employ the Iwasaki action for the gauge sector and the $N_{f}=$ 8 Domain-Wall action for the fermion. The Domain-Wall formalism we use is the same as the one originally proposed by Shamir in order to avoid complication related to its improvements. Throughout this study, the bare fermion mass is set to zero. The Domain-Wall height and the 5-th dimensional width are also fixed to $M=1.6$ and $L_{s}=12$, respectively. We set periodic boundary conditions in the spatial directions and an anti-periodic condition in the temporal direction. By using the IroIro++ code system [䧃], we generate gauge configurations with several values of $\beta$ on two different volumes $8^{3} \times 32$ and $16^{3} \times 64$. The unconventionally large size in the time direction is for a critical study of the meson propagation presented in Section 4 . In the molecular dynamics of HMC, we use the two time-step Omelyan integrator with the step size $\Delta \tau$ and $\Delta \tau / 4$ for pseudofermions and link variables, respectively. In Table $1, \Delta \tau$ and the number of thermalized trajectories are listed for each ensemble ( $\beta$ and the lattice volume). The acceptance rates are more than $90 \%$ for all generations.

Because of the massless simulation, slow thermalization and long auto-correlations are anticipated, especially for the large $\beta$ ensembles. We estimate these MD-times by monitoring the magnitude of spatial Polyakov loops $\left|P_{x}\right|,\left|P_{y}\right|$ and $\left|P_{z}\right|$ on the configurations. From a perturbative argument of the vacuum structure [3] , at the larger $\beta$ region, the spatial Polyakov will converge to a non-zero value (with some fluctuation) whereas the temporal ones remain around zero. We can exploit the condition that the spatial Polyakov loops converge to some non-zero values. Results from these rough estimation are listed in the third and fourth column of the tables. It is noted that the number of thermalized trajectories are several times larger than the auto-correlation length for all gauge ensembles. Therefore, the statistical localization can be avoided by measurements covering whole thermalized trajectories.

To check the chiral property of the generated configurations, we compute residual fermion mass $a m_{\text {res }}$ using the definition by the violation of the Ginsparg-Wilson relation [可. Simply averaging the measured values on a few sample configurations for each ensemble, we obtain typical magnitudes of $a m_{\text {res }}$ as listed in the last column. Those values are sufficiently small (i.e. $0.1 \%$ of the pseudo-scalar masses on the corresponding ensembles) to allow us to directly investigate the 

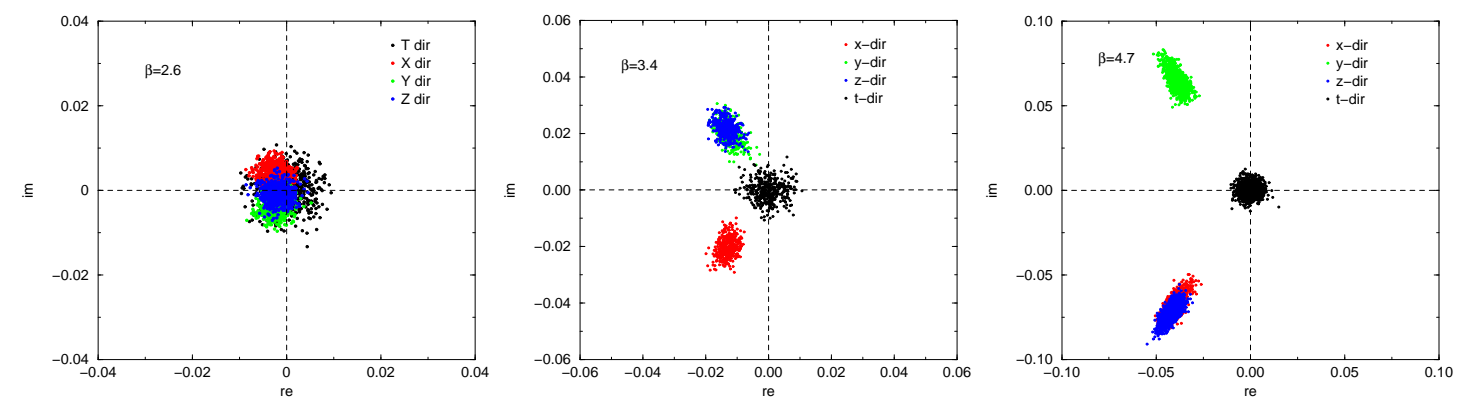

Figure 2: Distribution of the Polyakov loop measured on each gauge configurations at $\beta=2.6$ (left),3.4 (center) and 4.2 (right). In each panel, $P_{t}$ is shown by black symbols and $P_{x, y, z}$ by different colors. Results on the $16^{3} \times 64$ lattice are shown.
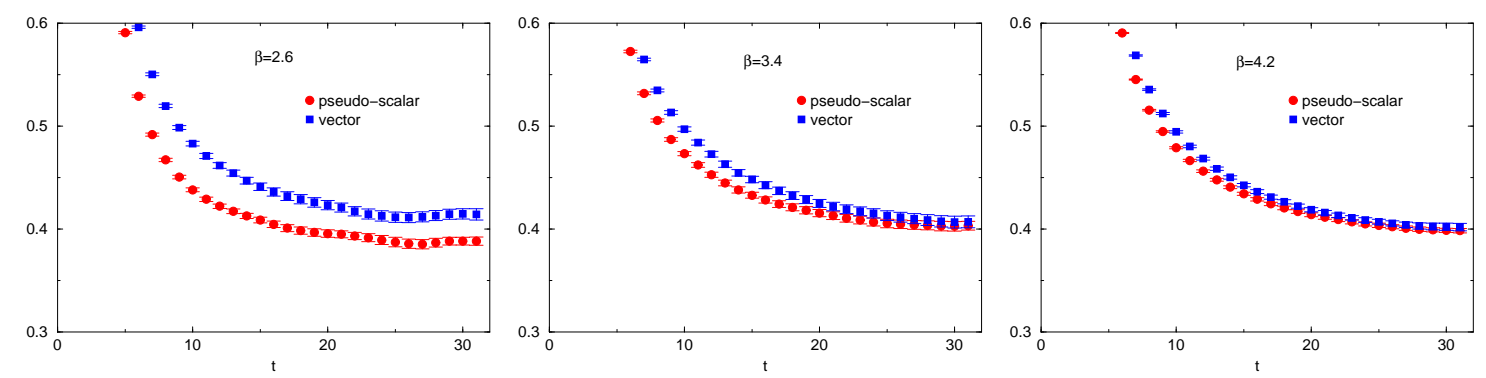

Figure 3: Effective masses of pseudo-scalar (red) and vector (blue) mesons at $\beta=2.6$ (left),3.4 (center) and 4.2 (right). Results on the $16^{3} \times 64$ lattice are shown.

properties of the conformal region in the diagram.

\section{Vacuum structure}

Figure 2 shows the distribution of Polyakov loops in the complex plane at different $\beta$ 's on the $16^{3} \times 64$ lattice. We observe that at $\beta=2.6$ the distribution is centralized at the origin, while at $\beta=3.4$ the Polyakov loops in the spatial directions take the angles $\pm 2 \pi / 3$, as in the previous works with the Wilson fermions [B]. At $\beta=4.2$, the distribution becomes sharper and the magnitude increases. Thus the $Z(3)$ symmetry is broken for $\beta \geq 3.4$ in the spatial directions, but the $Z(3)$ symmetry is preserved in the temporal direction. In other words, a phase transition from the $Z(3)$ symmetry symmetry phase to the broken phase seems to occur between $\beta=2.6$ and $\beta=3.4$.

The meson temporal propagators and masses contain useful and important information for the investigation of the properties of the vacuum structure. The correlation functions are computed in steps of 10 trajectories. We improve the signal by taking average of the propagator obtained with local sources at different time-slices in intervals of 8 for $\beta=2.6$ and 3.4 on the $16^{3} \times 64$ lattice, and of 4 for others. Three panels of Figure 3 show the effective masses obtained assuming the cosh function on the $16^{3} \times 64$ lattice. In each panel, we show the vector and pseudo-scalar channels at the same $\beta$ 's as Figure 2. The mass difference seen at $\beta=2.6$ diminishes at $\beta=3.4$ and disappears at even higher $\beta$. This is a sign of restoration of the chiral symmetry. Combined with the analysis 

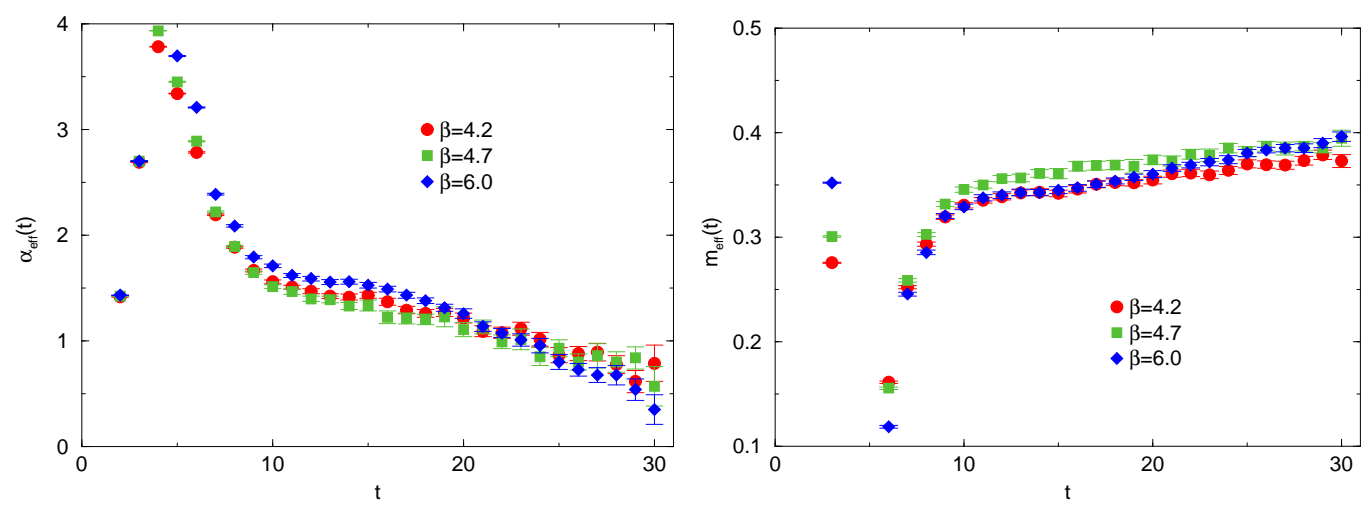

Figure 4: Effective values of $\alpha$ (left panel) and $m$ (right panel) on the $16^{3} \times 64$ lattice. Results at $\beta=4.2$ (red), 4.7 (green) and 6.0 (blue) are shown.

of the spatial Polyakov loops above the data suggest that there is a phase transition taking place between the confining phase and the conformal phase between $\beta=2.6$ and $\beta=3.4$.

We note that the data behavior described in this section is more distinct with the $16^{3} \times 64$ volume than $8^{3} \times 32$. This implies that data obtained in a even larger volume would allow us to investigate properties of the transition in more detail. In the following discussion, we assume our gauge ensembles at $\beta \geq 3.4$ on the $16^{3} \times 64$ are in the conformal region.

\section{Meson propagators}

Now we investigate the property of the conformal phase through the pseudo-scalar meson temporal propagator. It is known that in the conformal theory all correlation functions without any IR cutoff show a power law behavior, $\propto t^{-\alpha}$, at long distances. However, given our system is in a finite box, the propagation is influenced by the IR-cutoff. Ref. [B]] predicts that the modified behavior is $\propto t^{-\alpha} e^{-m t}$ ("the power-law corrected Yukawa type decay"), which looks a hybrid of the power law and the ordinary exponential decay, $\propto e^{-m t}$.

We parametrize the propagator as $G(t)=A t^{-\alpha} e^{-m t}$. Assuming a mild $t$-dependence of $\alpha$ and $m$ such that they do not significantly change between time slices at $t$ and $t \pm 1$, those effective values are obtained from the data of $G(t)$ and $G(t \pm 1)$. In the two panels of Figure 4, we plot those quantities on the $16^{3} \times 64$ lattice for $\beta=4.2,4.7$ and 6.0 with different colors. In the figure, the assumption of the mild $t$-dependence is reasonably satisfied while there is no significant $\beta$ dependence observed with the current statistics. Applying the relation $\alpha=3-2 \gamma *_{m}$ to the plat region $10 \leq t \leq 20$ for $\beta=4.2$, we obtain a rough estimation $0.2 \lesssim \gamma_{m} \lesssim 0.6$, which is consistent with the previous result [B] (see Eq. 11, pages 31 and 33). To increase the reliability of the estimate we will increase statistics at $\beta=3.4$, which is closer to the transition point. (We have excluded the data at $\beta=3.4$ in Figure 4 due to poor statistics.)

As another important analysis using the meson propagator, we attempt to determine the location of the infra-red fixed point (IRFP) [ [ $]$ ]. For the lattice size $N$, we consider the scaled propagator $\tilde{G}\left(\tau=t / N_{t} ; N\right)=G(t ; N)$. At IRFP, due to the finite size scaling, $\tilde{G}$ with the different lattice sizes 

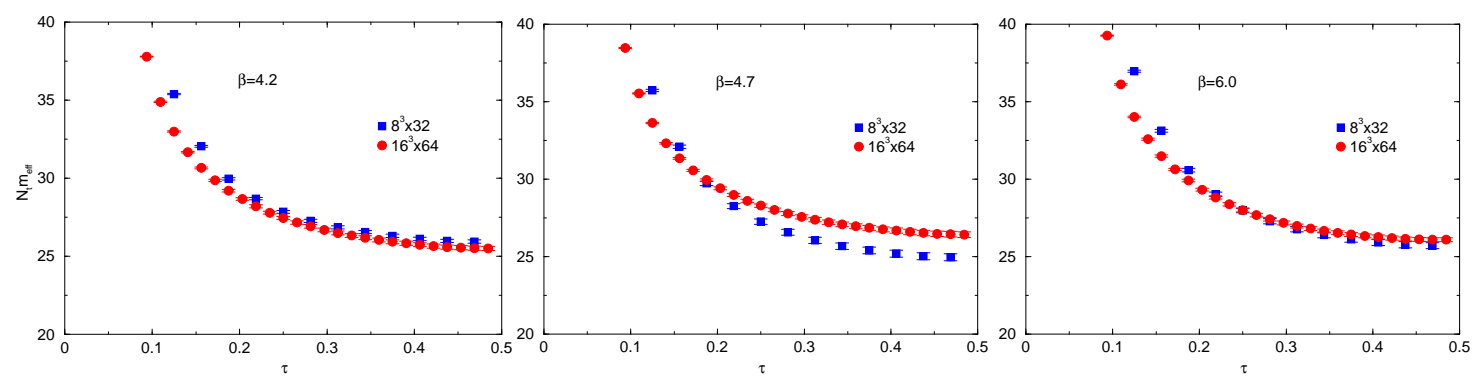

Figure 5: Comparison of the effective mass $\hat{m}$ obtained with two different volumes $16^{3} \times 64$ (red) and $8^{3} \times 32$ (blue) at $\beta=4.2$ (left), 4.7 (center) and 6.0 (right).

$N$ and $N^{\prime}$ are related by

$$
\tilde{G}(\tau ; N)=\left(N^{\prime} / N\right)^{3-2 \gamma_{m}} \tilde{G}\left(\tau ; N^{\prime}\right)
$$

This relation further implies that the scaled effective mass defined by

$$
\hat{m}(\tau ; N) \equiv N_{t} \ln (G(t ; N) / G(t+1 ; N))
$$

should be volume-independent at IRFP. In Figure 5, we compare $\hat{m}$ at three different $\beta$ 's in the conformal region on the two lattice volumes. In the figure, we observe the magnitude relation of the two volumes at large $\tau$ changes between $\beta=4.2$ and 4.7, which implies the existence of IRFP in this region. For a conclusive determination of the existence and the location of IRFP, we need more data points with different $\beta$ to narrow the $\beta$-region and those with larger volume to confirm the size-independence.

\section{Future plans}

Besides the analyses presented in the last two sections, we attempt to obtain spectral density $\rho(\lambda)$ of the Dirac-eigenvalue $\lambda$ by the stochastic method [Q, प⿺, ㅁ] ]. However, the discussion based on the Banks-Casher relation requires the large volume limit before the chiral limit is taken. Also, it should be noted that the sign of the chiral symmetry violation may be so delicate that a careful study as seen in ref. [ए2] is necessary. That means calculations have to be done in a sufficiently large volume with non-zero fermion masses to be able to determine whether or not the chiral symmetry is conserved in the conformal region. The same is valid to extract $\gamma_{m}$ in a reliable manner.

As seen in the plots presented in Section 3 and 4, the data of meson propagator have to be improved by more statistics. We are also planning to generate gauge ensembles on a larger volume to conclude our study. Combining the data on those ensembles with the current ones, it is possible to sort out the issues of the phase transition in Section 3 and of the property of the meson propagator in Section 4, as well as the above Dirac-spectrum analysis. A natural target is $32^{3} \times 128$. At the same time, we aim to understand the relation of our work with others which use different fermion formalism or setup [[1, [1], [4]]. 


\section{Acknowledgment}

Numerical simulations are performed on the IBM System Blue Gene Solution at High Energy Accelerator Research Organization (KEK) under a support of its Large Scale Simulation Program (No. 14/15-18). This work is supported in part by the Grant-in-Aid of the Japanese Ministry of Education (No. 15K05065) and the SPIRE (Strategic Program for Innovative Research) Field5 project.

\section{References}

[1] A. Hasenfratz, Beyond the Standard Model: lattice calculations at the energy frontier, in these proceedings.

[2] K.-I. Ishikawa, Y. Iwasaki, Y. Nakayama and Y. Yoshie, Conformal theories with an infrared cutoff, Phys. Rev. D 87 (2013) 071503.

[3] K.-I. Ishikawa, Y. Iwasaki, Y. Nakayama and Y. Yoshié, Global Structure of Conformal Theories in the SU(3) Gauge Theory, Phys. Rev. D 89 (2014) 114503.

[4] G. Cossu, J. Noaki, S. Hashimoto, T. Kaneko, H. Fukaya, P.A. Boyle and J. Doi, JLQCD IroIro++ lattice code on BG/Q, PoS(LATTICE 2013) 482, URL:

http://suchix.kek.jp/guido_cossu/documents/DoxyGen/html/in dex.html

[5] R. G. Edwards, B. Joo, A. D. Kennedy, K. Orginos, U. Wenger, Comparison of Chiral Fermion Methods, PoS(LATTICE 2005) 146.

[6] L. Del Debbio and R. Zwicky, Hyperscaling relations in mass-deformed conformal gauge theories, Phys. Rev. D 82 (2010) 014502.

[7] A. Cheng, A. Hasenfratz, G. Petropoulos and D. Schaich, Scale-dependent mass anomalous dimension from Dirac eigenmodes, JHEP 07 (2013) 061.

[8] K.-I. Ishikawa, Y. Iwasaki, Y. Nakayama and Y. Yoshié, IR fixed points in SU(3) gauge theories, Phys. Lett. B748 (2015) 289.

[9] L. Giusti and M. Lüscher, Chiral symmetry breaking and the Banks-Casher relation in lattice QCD with Wilson quarks, JHEP 03 (2009) 013.

[10] K. Holland, J. Kuti and C. H. Wong, A new method to calculate the Dirac operator spectral density, in these proceedings.

[11] S. Hashimoto, G. Cossu, H. Fukaya, T. Kaneko, J. Noaki, Stochastic calculation of the QCD Dirac operator spectrum with Möbius domain-wall fermion, in these proceedings.

[12] G. Cossu, H. Fukaya, S. Hashimoto and A. Tomiya, Violation of chirality of the Möbius domain-wall Dirac operator from the eigenmodes, [arXiv:1510.07395].

[13] Y. Aoki, et. al. (LatKMI Collab.), Walking signals in $N_{f}=8$ QCD on the lattice, Phys. Rev. D 87 (2013) 094511.

[14] Y. Aoki,et. al. (LatKMI Collab.), Light composite scalar in eight-flavor QCD on the lattice, Phys. Rev. D 89 (2014) 111502(R). 